\title{
Unresolved Problems in the Life Cycle of Truffles
}

\author{
Varda Kagan-Zur ${ }^{*}$ and Nurit Roth-Bejerano
}

Department of Life Sciences, Ben-Gurion University, P.O. Box 653, Beer Sheva 84105, Israel

\begin{abstract}
The mating system and ploidy level of truffles, both mycorrhizal and sterile fruit-body hyphae are the subject of conflicting hypotheses. The current dogma concerning Ascomycetes, supported by recent studies on truffles, maintains that the mycorrhizing mycelium is homokaryotic and that the short-lived ascogenous heterokaryotic hyphae resulting from the fertilization process occur only in truffle primordia. Therefore the gleba and sterile veins are also homokaryotic. Other studies show heterokaryons both in gleba and mantle hyphae of some truffle species, leading to the conclusion that longterm heterokaryons do exist in truffles. The matter is discussed.
\end{abstract}

Some fundamental aspects concerning the life cycle of truffles (Pezizales) [1] have yet to be fully elucidated. Among these are the mating system and the ploidy level of both mycorrhizal and sterile fruit-body hyphae. Both are the subject of conflicting hypotheses.

Tuber and Terfezia hyphal cells, like those of other Ascomycetes, are multinucleate (e.g. [2]). Ascertaining whether a mycelial culture is a homo or heterokaryon is, therefore, not straightforward. Nowadays, however, the prevailing perception, which is based on in-depth research, casts doubt on the long-term subsistence of natural vegetative heterokaryons in most filamentous Ascomycetes (e.g. [3]).

The hypothesis that tends to be accepted today - a hypothesis that has been thoroughly investigated - maintains that the vegetative mycelium of such fungi is homokaryotic and that plasmogamy occurs only in the primordia of the fruit bodies. Plasmogamy in the developing fruit body leads to the formation of heterokaryotic ascogenous hyphae, from which, after karyogamy and meiosis, haploid ascospores develop (e.g. [2]). As a result, the heterokaryotic phase is brief and is restricted to the developing ascocarps.

Yet, it had long been known that long-term heterokaryons exist in Neurospora crassa, Pezizomycotina [4]. This was later shown to be dependent on the existence of identical het alleles in the individual strains forming the heterokaryon [5]. Wild type Neurospora tetrasperma isolates are invariably heterokaryons, mostly selfing, but maintain some level of outcrossing in nature, as well as in the laboratory [6]. This phenomenon is also dependent on het allele identity in both nuclei forming the long-term heterokaryion (ibid). Thus conditions under which long term ascomyceteous heterokaryons may be maintained - do exist.

No het genes were, so far, identified or demonstrated in truffles. But Terfezia boudieri and Kalaharituber pfeilii heterokaryon mycelial cultures originating from glebal outgrowth seem to be the rule rather than exception: In glebal $K$. pfeilii and $T$. boudieri cultures, the hyphal cells invariably

*Address correspondence to this author at the Department of Life Sciences, Ben-Gurion University, P.O. Box 653, Beer Sheva 84105, Israel; Tel: + 972 8 6461338; Fax: +972 86472984 ;

E-mail: zur@bgu.ac.il or kaganzur@gmail.com contained paired nuclei [7]. K. pfeilii hyphae originating from single ascospore cultures (Pezizaceae are presumed to bear uninucleate spores; [8]) showed single, unpaired, nuclei, while hyphae obtained at the contact zone between two different single-spore-derived mycelia had paired nuclei similar to gleba derived cultures [7]. Similar findings have been reported for Morchella [9].

Moreover, one such paired-nuclei culture actually contained two different variants of the ITS (Internal Transcribed Spacer of the rRNA gene cluster) region in a single hypha. This culture has now been maintained for about 10 years without any alterations taking place in the (equal) intensity of the two variants [10]. Had the two ITS types been contained together within each nucleus in the culture, then according to the theory of molecular drive processes and concerted evolution $[11,12]$, one should at least have begun to overtake the other. Ganley and Scott [13] have demonstrated the rapid appearance (within two generations of single spore purification) of a range of ribosomal spacer heterogeneity following karyogamy in a hybrid fungal endophyte where each parent had a single and stable form of spacer; their conclusion was that unification of new forms might take place within a few years following karyogamy. The fact that such unification did not occur in the case of our T. boudieri ITS variants seems to support the possibility of long-term maintenance of the two forms in separate nuclei, i.e. a heterokaryotic culture [10].

It has consistently been assumed that the ascocarps of Tuber melanosporum and Tuber magnatum - the black Perigord truffle and the white Piedmont truffle, respectively - are also diploids (heterokaryotic?), and have therefore been regarded as selfing species. The reason is that when codominant markers were evaluated, heterozygous ascocarps were not detected [14-18]. This, in itself, may not be sufficient in view of their postglacial re-colonization patterns [18], but Poma et al. [19] studying Tuber aestivum demonstrated, by counting chromosomes, that hyphal cells had twice as many chromosomes as single spores, and, in view of the chromosome number, a single spore contained a single nucleus. It is not clear whether the hyphae studied were glebal or ascogenous. However, in a following review [20] they conclude that, as ectomycorrhizal mantle and ascocarpal cells contain, 
in general, two nuclei, and as asci contain a single one, the hypothesis that the primary mononucleate mycelium is followed by a dikaryotic infecting one seems to be confirmed. Consistent with these findings are the results of an old experiment [21], which suggested that monosporic mycelia could not form mycorrhizas while multisporic cultures could; meaning - heterokaryons form mycorrhiza, monokaryons do not. These studies support the notion of long-term heterokaryons in Tuber spp.

Contrary to the above, Paolocci et al. [22] and Rubini et al. [23] using microsatellite markers, demonstrated that some, and possibly considerable, outcrossing takes place in T. magnatum. According to the authors, the vegetative infecting mycelium is homokaryotic, originating from a single ascospore germination. In line with current dogma, they maintain that fertilization (plasmogamy, closely followed by karyogamy) occurs in the primordia of the ascocarps. They also maintain that the donor of the genetic complement could be asexual spores (conidia), recently detected on Tuber borchi and Tuber oligospermum mycelia [24] which may be assumed to exist in other truffle species as well. According to their hypothesis ascogenous heterokaryotic hyphae resulting from the fertilization process are short lived and surrounded by homokaryotic sterile maternal vegetative hyphae. Therefore the mature ascocarps are composed exclusively of asci and homokaryotic hyphae (gleba and sterile veins). Since as many as $75 \%$ of the supposedly single-spore mycorrhizal root tips were of purely maternal origin (the expected outcome was $25 \%$ ), considerable selfing probably occurred. May these results be interpreted in terms of the similar findings for $N$. tetrasperma mentioned above?

In a follow up paper Riccioni et al [25], while assessing T. melanosporum genetic variability, describe instances where T. melanosporum fruit bodies contained asci harboring different ITS or microsattelite haplotypes as compared to their corresponding gleba. The number of such fruit bodies was low (7 out of 58) in a population where a relatively high variability was encountered, and, since no ratio of paternal to maternal haplotypes was presented, the fraction of outcrossed asci remains obscure. If this ratio turns out to be similar to findings for T. magnatum, then, in view of the low number of fruit bodies harboring non-maternal asci, their finding would support a $N$. tetrasperma scenario.

Some T. boudieri and K. pfeilii fruit bodies were found to harbor two ITS forms each. As no glebal culture could be obtained from the dry fruit bodies of the latter, it is not clear whether the two were maintained in intertwined separate hyphae or, as in the T. boudieri double-profile culture described above, in separate nuclei sharing the same cytoplasm. Each germinated K. pfeilii ascospore, however, harbored only one of the two forms [26]. The mere existence of such heterozygous fruit bodies seems to contradict the notion of a solely non-hyphal genetic complement donor. In fact, Riccioni et al [25], encountered rare fruit bodies (3 out of over 200) harboring two forms of ITS each. They interpret this phenomenon in terms of distinct ITS copies maintained in a single rRNA cluster in one nucleus. However, the same result may be viewed in terms of each ITS type being maintained in a separate nucleus, i.e. a heterokaryon, or in terms of interwined hyphae, as argued above.
In addition, Paolocci et al. [22] and Rubini et al. [23] demonstrated that single mycorrhized root tips harbored only one combination of marker alleles, indicating that the mycorrhizing agent is a homokaryotic, monosporic mycelium. This is certainly compelling (though the use of microsatellites as markers could be viewed as problematic owing to the possibility of instability of microsattelite markers, especially during meiosis). Accordingly, although their claim is incompatible with the results discussed above [20,21], we cannot rule out the possibility that, for Tuber spp, each of the two homokaryons or heterokaryons - may inoculate host plant roots.

As to Pezizaceae - in our hands, long-term T. boudieri and $K$. pfeilii cultures, which were invariably derived from either glebal outgrowth (sterile hyphae) or multi-spore germination (K. pfeilii); and therefore heterokaryons, as discussed earlier, were successfully and repeatedly used in mycorrhization experiments [27-29]. In accordance with findings for T. melanosporum [21], we never obtained mycorrhizas when homokaryon cultures were employed [unpublished results]. However, both mono- and multisporic Terfezia arenaria inocula were reported to successfully mycorrhize host plants [30].

We, therefore, maintain that for all truffles and possibly other genera or families, a likely scenario consistent with the evidence would include the following stages: ascospores germinate to produce the primary homokaryotic mycelium. This mycelium develops in the soil, where it may either inoculate host plants as a primary homokaryotic mycelium, or produce a secondary heterokaryotic mycelium after undergoing plasmogamy (i.e. before inoculation). It is not known precisely when plasmogamy occurs in the case of inoculation by a primary mycelium, nor what is the identity of the fertilizing complement donor. However, fruit bodies seem to be formed by heterokaryotic hyphae, (gleba and sterile veins as well as fertile veins) as indicated by paired nuclei [7] and the existence of two nuclei in single mantle cells [20]. Karyogamy and meiosis probably take place within the fruit body primordia.

\section{REFERENCES}

[1] Laessoe T, Hansen K. Truffle trouble: what happened to the Tuberales. Mycol Res 2007; 111: 1075-99.

[2] Alexopoulos CJ, Mims CW, Blackwell M. Introductory mycology. 4th ed. New York: John Wiley\&Sons, Inc; 1996.

[3] Glass NL, Jacobson DJ, Shiu PKT. The genetics of hyphal fusion and vegetative incompatibility in filamentous ascomycete fungi. Annu Rev Genet 2000; 34: 165-86.

[4] Beadle GW, Coonradt VL. Heterocaryosis in Neurospora crassa. Genetics 1943; 29: 291-308.

[5] Pandit A, Maheshwari R. Demonstration of the role of het genes in heterokaryon formation in Neurospora under simulated field conditions. Fungal Genet Biol 1996; 20: 99-102.

[6] Powell AJ, Jacobson DJ, Natvig DO. Allelic diversity at the het-c locus in Neurospora tetrasperma confirm outcrossing in nature and reveals an evolutionary dilemma for pseudohomothallic Ascomycetes. J Mol Evol 2001; 52: 94-102.

[7] Roth-Bejerano N, Li Y-F, Kagan-Zur V. Homokaryotic and heterokaryotic hyphae in Terfezia. Anton Leeuw Int J 2004; 85: 165-8.

[8] Zhang B-C. Nuclear numbers in Geneaceae and Terfeziaceae ascospores and their taxonomic value. Syst Ascomycet 1992; 11: 31-5.

[9] Volk TJ, Leonard TJ. Cytology of the life cycle of Morchella. Mycol Res 1990; 94: 399-406. 
[10] Aviram S, Roth-Bejerano N, Kagan-Zur V. Two ITS forms coinhabiting a single genet of an isolate of Terfezia boudieri (Ascomycotina), a desert truffle. Anton Leeuw Int J 2004; 85: 169-174.

[11] Dover G. Molecular drive: a cohesive mode of species evolution. Nature 1982; 299: 111-7.

[12] Dover G. Molecular drive in multigene families: how biological novelties arise, spread and are assimilated. Trends Genet 1986; 2: $159-65$.

[13] Ganley ARD, Scott B. Extraordinary ribosomal spacer length heterogeneity in a Neotyphodium endophyte hybrid: Implications for concerted evolution. Genetics 1998;150: 1625-37.

[14] Bertault G, Raymond M, Berthomieu A, Callot G, Fernandez D. Trifling variation in truffles. Nature 1998; 394: 734.

[15] Bertault G, Rousset F, Fernandez D, et al. Population genetics and dynamics of the black truffle in a man-made truffle field. Heredity 2001; 86: 451-8

[16] Frizzi, G, Lalli G, Miranda M, Pacioni G. Intraspecific isozyme variability in Italian populations of the white truffle Tuber magnatum. Mycol Res 2001; 105: 365-9.

[17] Mello A, Murat C, Vizzini A, Gavazza V, Bonfante P. Tuber magnatum, a species of limited geographical distribution: its genetic diversity inside and outside a truffle ground. Environ Microbiol 2005; 7: 55-65.

[18] Murat C, Diez J, Luis P, et al. Polymorphism at the ribosomal DNA ITS and its relation to postglacial re-colonization routes of the Perigord truffle Tuber melanosporum. New Phytol 2004; 164: 401-11.

[19] Poma A, Pacioni G, Ranalli R, Miranda M. Ploidy and chromosomal number in Tuber aestivum. FEMS Microbiol Let 1998; 167: 101-5.
[20] Poma A, Limongi T, Pacioni G. Current state and perspectives of truffle genetics and sustainable biotechnology. Appl Microbiol Biotechnol 2006; 72: 437-41.

[21] Bonfante-Fasolo P, Brunel A. Caryological features in a mycorrhizal fungus: Tuber melanosporum Vitt. Allionia 1972; 18: 5-11.

[22] Paolocci F, Rubini A, Riccioni C, Arcioni S. Reevaluation of the Life Cycle of Tuber magnatum. Appl Environ Microbiol 2006; 72: 2390-3.

[23] Rubini A, Riccioni C, Arcioni S, Paolocci F. Troubles with truffles: unveiling more of their biology. New Phytol 2007; 174: 256-9.

[24] Urban A, Neuner-Plattner I, Krisai-Greilhuber I, Haselwandter K. Molecular studies on terricolous microfungi reveal novel anamorphs of two Tuber species. Mycol Res 2004; 108: 749-58.

[25] Riccioni C, Belfiori B, Rubini A, Passeri V, Arcioni S, Paolocci F. Tuber melanosporum outcrosses: analysis of the genetic diversity within and among its natural populations under this new scenario. New Phytol 2008; 179: DOI 10.1111/j.1469-8137.2008.02560.x.

[26] Kagan-Zur V, Kuang J, Tabak S, Taylor FW, Roth-Bejerano N. Potential verification of a host plant for the desert truffle Terfezia pfeilii by molecular methods. Myc Res 1999; 103: 1270-4.

[27] Roth-Bejerano N, Livne D, Kagan-Zur V. Helianthemum-Terfezia relations in different growth media. New Phytol 1990; 114: 235-8.

[28] Kagan-Zur V, Raveh E, Lischinsky S, Roth-Bejerano N. Helianthemum - Terfezia association is enhanced by low iron in the growth medium. New Phytol 1994; 127: 567-70.

[29] Zaretsky M, Kagan-Zur V, Mills D, Roth-Bejerano N. Analysis of mycorrhizal associations formed by Cistus incanus transformed root clones with Terfezia boudieri isolates. Plant Cell Rep 2006; 25: $62-70$.

[30] Fortas Z, Chevalier G. Carackristiques de la germination des ascospores de Terfezia arenaria Moris Trappe, recolte en Algerie. Cryptogamie Mycol 1992; 13: 21-9. 